\title{
Modification of Ceftibuten Transport by Changes in Lipid Fluidity Caused by Fatty Acid Glycerol Esters
}

\author{
Kenjiro Koga, ${ }^{*, a}$ Masahiro Murakami, ${ }^{b}$ and Susumu Kawashima ${ }^{c}$ \\ Division of Pharmaceutical Information, ${ }^{a}$ Department of Pharmaceutics, ${ }^{c}$ Faculty of Pharmaceutical Sciences, Hokuriku \\ University, Ho-3, Kanagawa-machi, Kanazawa 920-1181, Japan and Research and Development Department Amato \\ Pharmaceutical Products, Ltd., ${ }^{b}$ Fukuchiyama 620-0932, Japan. $\quad$ Received August 18, 1998; accepted October 23, 1998
}

\begin{abstract}
The effects of various fatty acid glycerol esters (FAGE) on the transport of ceftibuten in rat intestinal brushborder membrane vesicles (BBMV) were investigated. The HLB numbers of FAGE used were in the range of 6.5 to 15.7. The ceftibuten uptake by BBMV was examined by a rapid filtration method in the presence or absence of FAGE of a non-solubilizing concentration $(0.03 \%$, w/v). Tetraglycerol monostearate, hexaglycerol monostearate, hexaglycerol sesquistearate, hexaglycerol tristearate and decaglycerol tristearate were found significantly to enhance the ceftibuten uptake under an inward $\mathrm{H}^{+}$-gradient condition, while FAGE with oleate or laurate did not affect it. The extent of ceftibuten uptake with FAGE correlated with the HLB of the esters $(r=-0.90, p<0.001)$. To elucidate the mechanism of its enhancing action, the effects of FAGE on the membrane lipid fluidity were examined using the fluorescence probes diphenylhexatriene (DPH) and trimethylammonium diphenylhexatriene (TMA-DPH) which distribute in the hydrophobic inner and outer regions of the lipid layer, respectively. The increase in ceftibuten uptake induced by FAGE proved to correlate with an increase in fluidity of the outer lipid layer $(r=-0.83, p<0.003)$ but not the inner lipid layer of BBMV. Hence, the enhancing effect of FAGE with the lower HLB on the ceftibuten transport in the presence of the $\mathrm{H}^{+}$-gradient should, at least in part, be explained by the interaction with the outer lipid regions of BBMV.
\end{abstract}

Key words fatty acid glycerol ester; non-ionic surfactant; ceftibuten; membrane lipid fluidity; brush-border membrane

It is important with respect to safety that the possible effects of surfactants being utilized as solubilizers or emulsifiers for oral pharmaceuticals on carrier-mediated transports of nutrients and medications be investigated. Our previous study ${ }^{1)}$ demonstrated that the uptake of ceftibuten, a $\beta$-lactam antibiotic absorbed via a proton coupled peptide transporter in the intestinal tract, ${ }^{2,3)}$ is inhibited by the addition of fatty acid sucrose monoester under an inward $\mathrm{H}^{+}$-gradient condition. The extent of inhibition of the ceftibuten uptake proved to correlate with the degree of interaction between the sucrose monoester and the membrane lipid of the isolated intestinal brush-border membrane vesicles (BBMV). Such an interaction was detected as a decrease in the membrane fluidity represented by the fluorescence anisotropy of 1,6diphenyl-1,3,5-hexatriene (DPH) and 1-(4-trimethylammoniumphenyl)-6-phenyl-1,3,5-hexatriene (TMA-DPH) which distribute in the hydrophobic inner and outer regions of the lipid layer, respectively. ${ }^{4)}$

Fatty acid glycerol esters (FAGE) are non-ionic surfactants developed for oral pharmaceutical use. The aim of this study was to investigate the effects of FAGE on the ceftibuten transport in BBMV in relation to change in the membrane lipid fluidity. We found that FAGE with stearate enhanced the initial uptake in the presence of an inward $\mathrm{H}^{+}$-gradient more than in the absence of this gradient. The change in membrane transport is discussed with respect to hydrophobicity of FAGE and change in membrane lipid fluidity.

\section{MATERIALS AND METHODS}

Materials Ceftibuten was a gift from Shionogi Co. (Osaka, Japan). Hexaglycerol monooleate (MO-500), decaglycerol monooleate (MO-750), tetraglycerol monolaurate (ML-310), hexaglycerol monolaurate (ML-500), decaglycerol monolaurate (ML-750), tetraglycerol monostearate (MS-

* To whom correspondence should be addressed.
310), hexaglycerol monostearate (MS-500), hexaglycerol sesquistearate (SS-500), hexaglycerol tristearate (TS-500) and decaglycerol tristearate (TS-750) were donated by Sakamoto Chemicals Co. (Tokyo, Japan). DPH and TMADPH were purchased from Wako Pure Chemicals Ind. (Osaka). $2^{\prime}, 7^{\prime}$-Bis(carboxyethyl)-4(5)-carboxyfluorescein (BCECF) was from Dojindo Laboratories (Kumamoto, Japan). The BCA protein assay $\mathrm{kit}^{\mathbb{B}}$ was obtained from Pierce Chemical Co. (Chicago, IL, U.S.A.). All other chemicals were analytical reagent grade.

Preparation of BBMV BBMV was prepared from the small intestine of male Wistar rats $(220-250 \mathrm{~g})$ by the $\mathrm{MgCl}_{2}$ precipitation method. ${ }^{5)}$ The membrane was purified about 10 to 15 -fold to the crude mucosa by comparison of the specific activities of marker enzymes of BBM, alkaline phosphatase and sucrase. Prepared BBMV were suspended in $20 \mathrm{~mm}$ HEPES-Tris buffer, $\mathrm{pH} 7.5$, containing $100 \mathrm{~mm}$ Dmannitol and $100 \mathrm{~mm} \mathrm{KCl}$ (buffer $\mathrm{A}$ ) at a concentration of $10 \mathrm{mg}$ protein $/ \mathrm{ml}$.

Stability of BBMV Solubilization of BBMV with FAGE was estimated in terms of turbidity. ${ }^{6)}$ Change in turbidity of the suspension with FAGE (range $0.005-0.1 \%$ ) was assayed using a Hitachi spectrophotometer 100-20 at $450 \mathrm{~nm}$. The membrane permeability was evaluated by the leakage of $\mathrm{BCECF}$, an impermeable fluorescence dye, preloaded in $\mathrm{BBMV}^{7)}$ in the presence or absence of FAGE. The BCECFloaded $\mathrm{BBMV}$ was prepared by the incubation of isolated rat intestinal mucosa with $5 \mu \mathrm{M} \mathrm{BCECF}$ for $30 \mathrm{~min}$ at $37^{\circ} \mathrm{C}$, followed by centrifugation of $70000 \times \boldsymbol{g}$ for $15 \mathrm{~min}$ to remove the untrapped dye. The precipitate was then resuspended in buffer $\mathrm{A}$ at a final concentration of $2 \mathrm{mg}$ protein $/ \mathrm{ml}$. To 1.5 $\mathrm{ml}$ of the BCECF-loaded BBMV, $1.5 \mathrm{ml}$ of $0-0.1 \%$ FAGE or $0.5 \%$ Triton $\mathrm{X}-100$ was added. The mixture was then incubated for $10 \mathrm{~min}$ at $25^{\circ} \mathrm{C}$, followed by centrifugation of $27000 \times \mathbf{g}$ for $20 \mathrm{~min}$. The fluorescence intensity of the super-

(C) 1999 Pharmaceutical Society of Japan 
Table 1. Physicochemical Parameters of FAGE and their Effects on Ceftibuten Uptake by BBMV

\begin{tabular}{|c|c|c|c|c|c|c|c|c|}
\hline \multirow{3}{*}{ No. } & \multirow{3}{*}{$\begin{array}{l}\text { Product } \\
\text { Control }\end{array}$} & \multirow{3}{*}{$\begin{array}{r}\text { HLB } \\
-\end{array}$} & \multirow{3}{*}{$\begin{array}{c}\text { c.m.c. } \\
\text { (mg/l) }\end{array}$} & \multirow{3}{*}{$\begin{array}{l}\text { Viscosity } \\
\text { (cP) }\end{array}$} & \multicolumn{4}{|c|}{$\begin{array}{c}\text { Ceftibuten uptake } \\
(\mathrm{nmol} / \mathrm{mg} \text { protein at } 1 \mathrm{~min}, \% \text { of control) }\end{array}$} \\
\hline & & & & & \multicolumn{2}{|c|}{$\mathrm{pH}_{\mathrm{in}}=7.5, \mathrm{pH}_{\mathrm{out}}=7.5$} & \multicolumn{2}{|c|}{$\mathrm{pH}_{\mathrm{in}}=7.5, \mathrm{pH}_{\mathrm{out}}=5.5$} \\
\hline & & & & & $0.23 \pm 0.04$ & $(100)$ & $1.24 \pm 0.14$ & $(100)$ \\
\hline 1 & MO-500 & 12.2 & 19.9 & 0.881 & $0.25 \pm 0.03$ & (109) & $1.33 \pm 0.13$ & (106) \\
\hline 2 & MO-750 & 14.5 & 21.6 & 0.880 & $0.21 \pm 0.03$ & $(91)$ & $0.94 \pm 0.14$ & (76) \\
\hline 3 & ML-310 & 11.9 & 19.7 & 0.881 & $0.24 \pm 0.04$ & (104) & $1.44 \pm 0.17$ & (116) \\
\hline 4 & ML-500 & 13.8 & 23.1 & 0.882 & $0.27 \pm 0.03$ & $(117)$ & $1.31 \pm 0.12$ & (105) \\
\hline 5 & ML-750 & 15.7 & 26.6 & 0.883 & $0.24 \pm 0.04$ & (104) & $1.16 \pm 0.12$ & (93) \\
\hline 6 & MS-310 & 10.2 & 13.0 & 0.883 & $0.26 \pm 0.04$ & (113) & $1.78 \pm 0.22 *$ & (143) \\
\hline 7 & MS-500 & 12.2 & 20.4 & 0.882 & $0.29 \pm 0.04$ & (126) & $1.93 \pm 0.21 * *$ & (155) \\
\hline 8 & SS-500 & 10.1 & 28.3 & 0.881 & $0.30 \pm 0.05$ & $(130)$ & $2.09 \pm 0.24 * *$ & (168) \\
\hline 9 & TS-500 & 6.5 & 14.8 & 0.882 & $0.32 \pm 0.04 *$ & (139) & $2.45 \pm 0.22 * *$ & (197) \\
\hline 10 & TS-750 & 9.1 & 15.9 & 0.882 & $0.30 \pm 0.05$ & $(130)$ & $1.94 \pm 0.19^{*}$ & (156) \\
\hline
\end{tabular}

The HLB numbers of FAGE were calculated from Griffin's equation. The c.m.c. and viscosity of FAGE were determined at $25^{\circ} \mathrm{C}$ by the measurement of pyrene fluorescence intensity using a Hitachi spectrofluorometer $850^{21)}$ and a glass viscometer, respectively. The uptake experiment of ceftibuten by BBMV was performed in the absence and presence of an inward $\mathrm{H}^{+}$-gradient condition at $25^{\circ} \mathrm{C}$. Uptake buffer was $20 \mathrm{~mm}$ HEPES-Tris (pH 7.5) or $20 \mathrm{~mm}$ MES-Tris (pH 5.5) containing $100 \mathrm{~mm}$ D-mannitol and $100 \mathrm{~mm} \mathrm{KCl}$. Uptake data represent the mean \pm S.D. of three to five experiments. Each FAGE concentration was $0.03 \%(w / v)$. *, $p<0.05 ; * *, p<0.01$ ( $v s$. control by Dunnett multiple range test).

natant was measured at $E_{\mathrm{x}}=490 \mathrm{~nm}$ and $E_{\mathrm{m}}=526 \mathrm{~nm}$ using a Hitachi spectrofluorometer 850 (Tokyo, Japan) at $25^{\circ} \mathrm{C}$. The release ratio of $\mathrm{BCECF}, f$, is defined as

$$
f=\left(f_{\mathrm{s}}-f_{\mathrm{b}}\right) /\left(f_{\mathrm{a}}-f_{\mathrm{b}}\right)
$$

where $f_{\mathrm{s}}$ is the fluorescence intensity of BCECF in BBMV with FAGE, $f_{\mathrm{b}}$ is the fluorescence intensity in BBMV without FAGE, and $f_{\mathrm{a}}$ is the fluorescence intensity in BBMV thoroughly dissolved by $0.5 \%$ Triton X-100.

Uptake Studies in BBMV The ceftibuten uptake study was carried out by the rapid filtration method using a Millipore filter (HAWP, $0.45 \mu \mathrm{m}, 25 \mathrm{~mm}$ diameter, Japan Millipore Ltd., Tokyo, Japan) in the absence or presence of an inward $\mathrm{H}^{+}$-gradient condition $\left(\mathrm{pH}_{\mathrm{in}}=7.5, \mathrm{pH}_{\text {out }}=7.5\right.$ or 5.5) at $25^{\circ} \mathrm{C}{ }^{8)}$ The reaction was initiated by the addition of $110 \mu \mathrm{l}$ of $20 \mathrm{~mm}$ HEPES-Tris buffer, $\mathrm{pH} 7.5$, or $20 \mathrm{~mm} 2-(\mathrm{N}$-morpholino)ethanesulfonic acid (MES)-Tris buffer, $\mathrm{pH} 5.5$, containing $100 \mathrm{~mm}$ D-mannitol, $100 \mathrm{~mm} \mathrm{KCl,} 0.6 \%$ dimethyl sulfoxide, $0.06 \%$ FAGE and $1.5 \mathrm{~mm}$ ceftibuten to $110 \mu \mathrm{l}$ of BBMV suspension. The extent of ceftibuten uptake into the BBMV was determined at $0.5,1,2,5$ and 20 min of incubation. The maximum uptake was generally observed at $1 \mathrm{~min}$ (data not shown). Therefore, the ceftibuten uptake in the presence of FAGE was compared with that in the absence of FAGE at $1 \mathrm{~min}$ after incubation. Ceftibuten was determined by the method of Sugawara et al. ${ }^{9)}$

Fluorescence Studies The fluorescence anisotropy of DPH and TMA-DPH reflects the fluidity of the inner (hydrophobic core domain) and outer (superficial domain) membrane lipid bilayer, respectively. ${ }^{4)} \mathrm{DPH}$ - or TMA-DPH-labeled membrane vesicles were prepared by the method described previously. ${ }^{10)}$ Fluorescence measurements were performed at $25^{\circ} \mathrm{C}$ in the presence or absence of $0.03 \%$ FAGE using a Hitachi spectrofluorometer 850 equipped with a rhodamine $\mathrm{B}$ quantum counter. The excitation and emission wavelengths in fluorescence measurements were 363 and $428 \mathrm{~nm}$ for DPH, 365 and $428 \mathrm{~nm}$ for TMA-DPH, respectively. The fluorescence anisotropy $(\gamma)$ was calculated using the following equation described by Ohyashiki et al., ${ }^{11}$

$$
\gamma=\left(I_{\mathrm{V}}-I_{\mathrm{H}}\right) /\left(I_{\mathrm{V}}+2 I_{\mathrm{H}}\right)
$$

where $I_{\mathrm{V}}$ and $I_{\mathrm{H}}$ represent the fluorescence intensities of the vertically and the horizontally polarized excitation, respectively. A $\Delta \gamma$ value represents the difference in the fluorescence anisotropy in the presence and absence of FAGE.

\section{RESULTS AND DISCUSSION}

Effects of FAGE on the Stability of BBMV We first examined the stability of BBMV in the presence of various concentrations of FAGE to determine the ester concentration where the integrity of the membrane was maintained. The turbidity of the BBMV suspensions decreased depending on the FAGE concentrations and the decrement in turbidity proved to relate to the HLB, probably hydrophobicity, of the ester used. The turbidity was more than $90 \%$ of the initial and the $f$ value of BCECF was less than 0.1 in the presence of less than $0.03 \%$ of FAGE. These results indicate that the integrity of BBMV is maintained under the experimental conditions. FAGE were used at the concentration of $0.03 \%$ in subsequent studies.

Effects of FAGE on Ceftibuten Uptake by BBMV As shown in Table 1, ceftibuten uptake in BBMV was significantly enhanced by the addition of FAGE with stearate (MS310 , MS-500, SS-500, TS-500 or TS-750) under the $\mathrm{H}^{+}$-gradient condition. The increased uptake was not observed in the absence of the $\mathrm{H}^{+}$-gradient except TS-500. The trapping of BBMV onto a Millipore filter in the presence of FAGE might also be affected by a possible change in viscosity of the uptake solution. However, the changes in the viscosity by the addition of FAGE were extremely small and almost negligible (Table 1). Consequently, these results suggest that FAGE may not affect the passive diffusion but the carrier-mediated transport of ceftibuten in BBMV.

Since a remarkably increased uptake of ceftibuten was observed by the addition of FAGE with stearate, we examined the relationship between hydrophobicity of FAGE and ceftibuten uptake. The correlation coefficient between the ceftibuten uptake and the HLB numbers of FAGE was -0.90 $(p=0.0003)$, while no correlation was seen between the critical micelle concentration (c.m.c.) values of FAGE and the uptake. These results suggest that FAGE must strongly inter- 


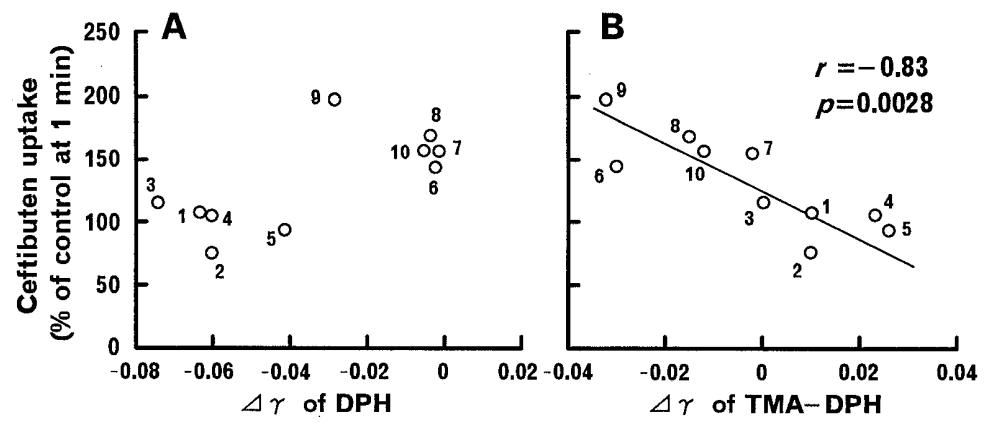

Fig. 1. Relationship between Ceftibuten Uptake by BBMV and the Fluorescence Anisotropy of DPH (A) or TMA-DPH (B) Labeled Membranes

The conditions for ceftibuten uptake and the fluorescence experiments are described under "Materials and Methods." The fluorescence anisotropy of DPH and TMA-DPH in the absence of FAGE was 0.250 and 0.285 , respectively. Standard deviation bars from the means for fluorescence anisotropy were less than 0.005 . Each FAGE concentration was $0.03 \%$ $(\mathrm{w} / \mathrm{v})$. Numbers indicate the chemical name of FAGE shown in Table 1.

act with the membranes depending on their hydrophobicity. As one possible mechanism, FAGE may affect the ceftibuten uptake by interaction such as binding ${ }^{12)}$ to and/or perturbation ${ }^{13)}$ of membranes. It was not learned in this study whether the enhanced transport induced by FAGE was based on a direct or indirect effect on its carrier proteins.

Effects of FAGE on Membrane Lipid Fluidity The carrier-mediated transport of drugs or nutrients was reportedly affected by the increase in membrane fluidity with the addition of the membrane mobility-enhancing agent 2-(2-methoxyethoxy)-ethyl-8-(cis-2-n-octylcyclopropyl) octanate. ${ }^{14,15)}$ It was therefore examined whether or not the increase of ceftibuten uptake induced by FAGE was related to the change in membrane fluidity of BBMV.

The relationship between the ceftibuten uptake and the fluorescence anisotropy of DPH or TMA-DPH in BBMV was evaluated in the presence of FAGE (Fig. 1). While no correlation was observed between the uptake and the $\Delta \gamma$ of DPHlabeled membranes, a high correlation was seen between the uptake and the $\Delta \gamma$ of TMA-DPH-labeled membranes ( $r=$ $-0.83, p=0.0028)$. These results were in good agreement with our previous observation that the ceftibuten uptake was decreased by the addition of fatty acid sucrose monoester which increased the fluorescence anisotropy of TMA-DPH in BBMV. ${ }^{1)}$ Taking these data together, the correlation between ceftibuten uptake and change in TMA-DPH anisotropy becomes higher with $r=-0.91(p=0.0001)$. The stearate-type FAGE that significantly enhanced ceftibuten uptake in BBMV decreased the $\Delta \gamma$ of TMA-DPH-labeled membranes but did not affect those of DPH-labeled membranes. FAGE with oleate (MO-500 and MO-750) or laurate (ML-310, ML500 and ML-750), on the other hand, strongly decreased the anisotropy of DPH, but not that of TMA-DPH. These results suggest that the enhancement effects of FAGE on ceftibuten uptake should be based on the interaction between FAGE molecules and membrane lipids in the hydrophobic outer leaflet regions of the membranes. Therefore, we propose that FAGE with stearate cause an increase in the fluidity of the hydrophobic superficial domain of BBMV on the basis of the hydrophobic interaction with membranes, thereby increasing permeability to ceftibuten. This proposed mechanism of increased membrane fluidity and enhanced ceftibuten uptake by the addition of FAGE may have potentially important implications for the mechanism of ceftibuten transport. Namely, it was predicted that the region of the membranes that en- hanced the transport capability of ceftibuten would be specific, because the hydrophobic interaction differs depending on the region of membrane lipid bilayers, and it is known that biological membranes adjust their physical properties for optimal functioning of membrane proteins and transporters. ${ }^{16)}$ Thus, membrane perturbation-increasing agents may result in modification with the transport capability of ceftibuten.

Muranishi ${ }^{17)}$ and Muranushi et al ${ }^{18)}$ proposed the perturbation theory of intestinal brush-border membrane lipids to explain the permeation enhancing mechanisms of mixed micelles on poorly permeable drugs. They concluded that the action of the mixed micelles was associated with the interaction of the incorporated lipid with the polar head group of phospholipid. The proton gradient-dependent ceftibuten transport in BBMV appears to be modified as a response to the perturbation of membrane lipids with the addition of FAGE.

We emphasize, however, that the relationship between the ceftibuten uptake and the inner or outer lipid fluidity of membranes was not paralleled by the addition of FAGE. FAGE that increased only the outer lipid fluidity of membranes appeared to improve the efficiency of ceftibuten transport. In addition, changes in fluidity of the hydrophobic core domain may not affect this transport. Our findings suggest that such changes in ceftibuten uptake induced by FAGE may account, at least in part, for an increase in the fluidity of the hydrophobic outer membranes. One possible proposed mechanism could be that FAGE with stearate appear to possess capabilities of incorporation into the superficial domains of the membrane to some extent, thereby increasing membrane fluidity, and the increased membrane fluidity may affect the transport activity of ceftibuten in the outer leaflet of the membranes, thereby enhancing membrane permeability.

Recent studies have demonstrated that P-glycoproteinmediated active efflux of drugs towards the luminal side of the intestine is inhibited by non-ionic surfactants such as Polysorbate 80 and Cremophor EL, although these surfactants are not likely to be the substrates for the efflux pumps. ${ }^{19,20)}$ Provided that ceftibuten can be transported towards the luminal side by the efflux pump, the esters which enhanced its uptake may result in interference with its drug transport capability. These complicated effects of surfactants on the intestinal transport require further investigation.

Acknowledgments We would like to thank Sakamoto 
Chemicals Co. (Tokyo, Japan) for the donation of FAGE. This work was supported in part by the Special Research Fund of Hokuriku University.

\section{REFERENCES}

1) Koga K., Murakami M., Kawashima S., Biol. Pharm. Bull., 21, 747 751 (1998)

2) Naasani I., Sato K., Iseki K., Sugawara M., Kobayashi M., Miyazaki K., Biochim. Biophys. Acta, 1231, 163-168 (1995).

3) Yoshikawa T., Muranushi N., Yoshida M., Oguma T., Hirano K., Yamada H., Pharm. Res., 6, 302-307 (1989).

4) Kitagawa S., Matsubayashi M., Kotani K., Usui K., Kametani F., J. Membr. Biol., 119, 221-227 (1991).

5) Molitoris B. A., Simon F. R., J. Membr. Biol., 83, 207--215 (1985).

6) Ohyashiki T., Takeuchi M., Mohri T., J. Biochem. (Tokyo), 95, 881-886 (1984).

7) Thwaites D. T., Brown C. D. A., Hirst B. H., Simmons N. L., J. Biol. Chem., 268, 7640-7642 (1993).

8) Sugawara M., Iseki K., Miyazaki K., J. Pharm. Pharmacol., 43, 433435 (1991)

9) Sugawara M., Toda T., Iseki K., Miyazaki K., Shirota H., Kondo Y., Uchino J.-I., J. Pharm. Pharmacol., 44, 968 -972 (1992).
10) Koga K., Murakami M., Kawashima S., Biol. Pharm. Bull., 20, 674 679 (1997).

11) Ohyashiki T., Sakata N., Matsui K., J. Biochem. (Tokyo), 111, $419-$ 423 (1992).

12) Maire M. L., Moller J. V., Champeil P., Biochemistry, 26, $4803-4810$ (1987).

13) Andersen J. P., Maire M. L., Kragh-Hansen U., Champeil P., Moller J. V., Eur. J. Biochem., 134, 205-214 (1983).

14) Drori S., Eytan G. D., Assaraf Y. G., Eur. J. Biochem., 228, 1020 1029 (1995).

15) Dudeja P. K., Wali R. K., Harig J. M., Brasitus T. A., Am. J. Physiol., 260, G586-G594 (1991).

16) Hui S. W., Sen A., Proc. Natl. Acad. Sci. U.S.A., 86, 5825-5829 (1989).

17) Muranishi S., Pharm. Res., 2, 108-118 (1985).

18) Muranushi N., Takagi N., Muranishi S., Sezaki H., Chem. Phys. Lipids, 28, 269-279 (1981).

19) Nerurkar M. M., Burton P. S., Borchardt R. T., Pharm. Res., 13, 528534 (1996)

20) Woodcock D. M., Jefferson S., Linsenmeyer M. E., Crowther P. J., Chojnowski G. M., Williams B., Bertoncello I., Cancer Res., 50, 4199-4203 (1990).

21) Ollmann M., Schwarzmann G., Sandhoff K., Galla H. J., Biochemistry, 26, 5943-5952 (1987). 